\title{
Why and how do teeth come off? -New insights into the tooth loss during periodontitis-
}

\author{
Takehito Ono ${ }^{1,2 *}$ \\ ${ }^{1}$ Department of Cell Signaling, Graduate School of Medical and Dental Sciences, Tokyo Medical and Dental University (TMDU), Yushima 1-5-45, Bunkyo-ku, \\ Tokyo, 113-8549, Japan \\ ${ }_{2}^{2}$ Japan Agency for Medical Research and Development, Core Research for Evolutional Science and Technology (AMED-CREST), Yushima 1-5-45, Bunkyo-ku, \\ Tokyo, 113-8549, Japan
}

Inadequate toothbrushing leads to the accumulation of dental plaque, which contains enormous number of bacteria inside. These bacteria stimulate immune cells to elicit inflammation in the periodontium, periodontitis. Periodontitis is the most common infectious disease and a major cause of tooth loss [1]. Because patients with multiple tooth loss suffer from mastication disorder and are subsequently exposed to a higher risk of aspiration pneumonia and dementia [2], dentists make every effort to preserve teeth. To date, there has been a substantial development in the treatment procedures of periodontitis [3-5].

In the periodontium under inflammation, there is an infiltration of many types of immune cells and these cells produce a variety of inflammatory cytokines [6]. Some of these cytokines are known to induce the expression of receptor activator of NF- $\kappa B$ ligand (RANKL), the essential cytokine for osteoclastogenesis $[7,8]$. Thus, it is widely accepted that bacteria induce inflammatory bone loss and subsequent tooth loss. However, the reason why and the mechanism of how teeth come off in the context of periodontitis have not been sufficiently addressed.

Recently, Tsukasaki, et al. demonstrated that a special IL-17producing $\mathrm{T}$ cell subset plays a crucial role in eradication of bacteria and alveolar bone resorption in a mouse model of periodontitis [9]. In the process of periodontitis, the number of $\mathrm{T}_{\mathrm{H}} 17$ cells derived from Foxp3 $3^{+}$Treg cells (exFoxp3 $\mathrm{T}_{\mathrm{H}} 17$ cells) and the number IL-17producing $\mathrm{T}$ cells showed a robust increase. In mice deficient in IL-17, there was an increase in bacterial load and alveolar bone resorption decreased. In mice deficient in $\alpha \beta \mathrm{T}$ cells, alveolar bone resorption also decreased. On the other hand, injection of exFoxp $3 \mathrm{~T}_{\mathrm{H}} 17$ cells was shown to exacerbate alveolar bone loss, indicating the contribution of them to bone loss in periodontitis. Conversion of Treg cells to exFoxp3 $\mathrm{T}_{\mathrm{H}} 17$ cells is known to be mediated by IL- 6 . The expression of this cytokine was also significantly upregulated after the induction of periodontitis. Its blockade or deficiency led to the decrease of the bone loss.

Bone resorption is mediated by osteoclast, the differentiation of which is induced by RANKL. RANKL is expressed by various types of cells including lymphocytes ( $\mathrm{T}$ cells and B cells) and mesenchymal cells such as bone marrow stromal cells, osteoblasts and osteocytes [8]. Because exFoxp $3 \mathrm{~T}_{\mathrm{H}} 17$ cells are known to highly express RANKL to drive osteoclastogenesis and IL-17 induce RANKL expression on mesenchymal cells $[10,11]$, the source of RANKL during bone loss in periodontitis was investigated. As a result, osteoblasts and periodontal ligament cells have turned out to be key sources of RANKL in periodontitis. RANKL adopts two forms (membrane-bound and soluble) and their physiological roles have been discussed for long [8]. Using a novel mouse strain which is deficient only in soluble form of RANKL but not in membrane-bound form [12], it was shown that membrane-bound form has a crucial role in inducing bone resorption in periodontitis. These results taken together clarified the mechanism of how periodontal inflammation occurs.

What is the reason for the tooth loss by periodontitis? Is it only a secondary effect of inflammation? Gingiva is the only region of the body where the continuity of epithelium is interrupted in the physiological condition, thus forming an entry site of bacteria into the periodontium, and tooth loss leads to the closure of the epithelial gap. Therefore, it is speculated that infection-induced tooth loss would save the body from the progression of infection. Extraction of periodontitis-affected teeth alleviated systemic infection, supporting this hypothesis.

This article revealed that, in periodontitis, $\mathrm{T}_{\mathrm{H}} 17$ cells converted from Foxp $3^{+}$cells protect the body by both eradication of pathogens and blocking the entry site. These cells have been previously shown to be a crucial arthritogenic $\mathrm{T}$ cell subset [11]. The effector cytokine of $\mathrm{T}_{\mathrm{H}} 17$ cells, IL-17, is reported to have both catabolic and anabolic effects on bone [13-15]. It seems interesting to note that these cells and cytokines can exert both destructive and protective effects depending on the situations they function. It is also noteworthy that this article has defined tooth loss as a termination of infection; otherwise it would remain to be regarded as mere a secondary effect of inflammation. Provided with a clearer view of tooth loss by periodontitis, we will be able to have a novel therapeutic strategy for periodontitis in the future, targeting exFoxp $3 \mathrm{~T}_{\mathrm{H}} 17$ cells and related cytokines to control both microbial infection and bone resorption.

${ }^{*}$ Correspondence to: Takehito Ono, Department of Cell Signaling, Graduate School of Medical and Dental Sciences, Tokyo Medical and Dental University (TMDU); Japan Agency for Medical Research and Development, Core Research for Evolutional Science and Technology (AMED-CREST) Yushima 1-5-45, Bunkyo-ku, Tokyo, 113-8549, Japan, Tel: +81-3-5803-5472; E-mail: ono.csi@ tmd.ac.jp

Received: March 04, 2018; Accepted: March 27, 2018; Published: March 30, 2018 


\section{References}

1. Tonetti MS, Jepsen S, Jin L, Otomo-Corgel J (2017) Impact of the global burden of periodontal diseases on health, nutrition and wellbeing of mankind: A call for global action. J Clin Periodontol 44: 456-462. [Crossref]

2. Fukushima-Nakayama Y, Ono T, Hayashi M, Inoue M, Wake H, et al. Reduced mastication impairs memory function. J Dent Res 96: 1058-1066. [Crossref]

3. Arweiler NB, Auschill TM, Sculean A (2018) Patient self-care of periodontal pocket infections. Periodontol 2000 76: 164-179. [Crossref]

4. Graziani F, Karapetsa D, Mardas N, Leow N, Donos N (2018) Surgical treatment of the residual periodontal pocket. Periodontology 2000 76: 150-163. [Crossref]

5. Preshaw PM (2018) Host modulation therapy with anti-inflammatory agents. Periodontol 2000 76: 131-149. [Crossref]

6. Hajishengallis G (2014) Immunomicrobial pathogenesis of periodontitis: keystones, pathobionts, and host response. Trends Immunol 35: 3-11. [Crossref]

7. Okamoto K, Nakashima T, Shinohara M, Negishi-Koga T, Komatsu N, et al. (2017) Osteoimmunology: The conceptual framework unifying the immune and skeletal systems. Physiol Rev 97: 1295-1349. [Crossref]
8. Ono T, Nakashima T (2018) Recent advances in osteoclast biology. Histochem Cell Biol. [Crossref]

9. Tsukasaki M, Komatsu N, Nagashima K, Nitta T, Pluemsakunthai W, et al. (2018) Host defense against oral microbiota by bone-damaging T cells. Nat Commun 9: 701. [Crossref]

10. Kotake S, Udagawa N, Takahashi N, Matsuzaki K, Itoh K. et al. (1999) IL-17 in synovial fluids from patients with rheumatoid arthritis is a potent stimulator of osteoclastogenesis. J Clin Invest 103: 1345-1352. [Crossref]

11. Komatsu N, Okamoto K, Sawa S, Nakashima T, Oh-hora M, et al. (2014) Pathogenic conversion of Foxp3+ T cells into TH17 cells in autoimmune arthritis. Nat Med 20 62-68. [Crossref]

12. Nagashima K, Sawa S, Nitta T, Tsutsumi M, Okamura T, et al. (2017) Identification of subepithelial mesenchymal cells that induce IgA and diversify gut microbiota. Nat Immunol 18: 675-682. [Crossref]

13. Ishigame $H$ (2009) Differential roles of interleukin-17A and $-17 \mathrm{~F}$ in host defense against mucoepithelial bacterial infection and allergic responses. Immunity 30: 108-119.

14. Ono T, Okamoto K, Nakashima T, Nitta T, Hori S, et al. (2016) IL-17-producing $\hat{I}^{\hat{P}^{\prime}} \hat{I}^{\prime} \mathrm{T}$ cells enhance bone regeneration. Nat Commun 7: 10928. [Crossref]

15. Uluçkan Ö, Jimenez M, Karbach S, Jeschke A, Graña O, et al. (2016) Chronic skin inflammation leads to bone loss by IL-17-mediated inhibition of Wnt signaling in osteoblasts. Sci Transl Med 8: 330ra337. [Crossref]

Copyright: (C2018 Ono T. This is an open-access article distributed under the terms of the Creative Commons Attribution License, which permits unrestricted use, distribution, and reproduction in any medium, provided the original author and source are credited. 